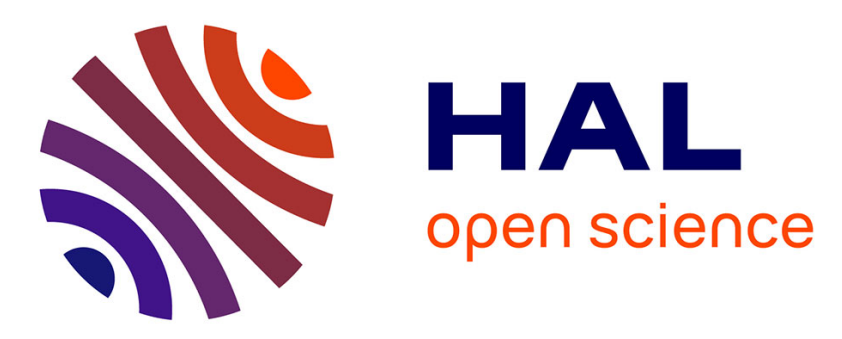

\title{
Energy relaxation in the spin glass AuFe $4 \%$ at low temperatures
}

\author{
A. Berton, J. Chaussy, J. Odin, R. Rammal, J. Souletie, R. Tournier
}

\section{To cite this version:}

A. Berton, J. Chaussy, J. Odin, R. Rammal, J. Souletie, et al.. Energy relaxation in the spin glass AuFe $4 \%$ at low temperatures. Journal de Physique Lettres, 1979, 40 (15), pp.391-394. 10.1051/jphyslet:019790040015039100 . jpa-00231651

\section{HAL Id: jpa-00231651 https://hal.science/jpa-00231651}

Submitted on 1 Jan 1979

HAL is a multi-disciplinary open access archive for the deposit and dissemination of scientific research documents, whether they are published or not. The documents may come from teaching and research institutions in France or abroad, or from public or private research centers.
L'archive ouverte pluridisciplinaire HAL, est destinée au dépôt et à la diffusion de documents scientifiques de niveau recherche, publiés ou non, émanant des établissements d'enseignement et de recherche français ou étrangers, des laboratoires publics ou privés. 


\title{
Energy relaxation in the spin glass $\underline{\mathrm{AuFe}} 4 \%$ at low temperatures $(*)$
}

\author{
A. Berton, J. Chaussy, J. Odin, R. Rammal, J. Souletie and R. Tournier \\ Centre de Recherches sur les Très Basses Températures, C.N.R.S., B.P. 166X, 38042 Grenoble Cedex, France
}

(Reçu le 18 mai 1979, révisé le 11 juin 1979, accepté le 18 juin 1979)

\begin{abstract}
Résumé. - Nous présentons les résultats de mesure du flux énergétique qui accompagne la relaxation de l'aimantation rémanente dans un verre de spins maintenu en situation isotherme. Ces résultats montrent que les barrières qui contrôlent cette relaxation vers l'état d'équilibre, séparent des niveaux d'énergie différents. Nous montrons qu'il est possible de relier les variations du flux énergétique à celles de l'aimantation rémanente.
\end{abstract}

\begin{abstract}
We present measurements of the energy flux associated with the relaxation of the remanent magnetization in a spin glass at constant temperature. The data show that the barriers which control this relaxation separate two levels with different energies. We show that it is possible to link the variations of the energy flux with those of the remanent magnetization.
\end{abstract}

1. Introduction. - The low temperature dynamical properties of spin glasses are currently the object of much interest [1]. Experimentally relaxation phenomena have been observed in spin glasses since quite a long time [2] (remanent magnetization, magnetization jumps, etc...). A characteristic feature is the wide range in time memories involved in these phenomena as is apparent in the logarithmic decrease of the remanent magnetization with time. Such aspects of the spin glass problem are shared with other disordered systems (glasses, polymers, etc...) where relaxation effects are also well characterized experimentally [3].

In this letter, we present a study of the isothermal relaxation of the energy in a spin glass at $T<T_{\mathrm{g}}$. Our results are interpreted within the frame of the standard two level systems model which has been widely used in the analysis of the experimental results of disordered substances. This allows a qualitative comparison to be made with the behaviour of glasses and polymers at low temperatures and below the vitreous transition.

2. Experimentals. - The sample is connected through a thermal link with a helium bath at $1.4 \mathrm{~K}$. It is possible to stabilize the temperature of the

(*) This work is a part of the thesis of J. O. who is also associated with «le laboratoire d'Electrotechnique», INPG, ERA 534 of C.N.R.S. English translation of a « note à l'Académie des Sciences ». sample at fixed values between $1.5 \mathrm{~K}$ and $5 \mathrm{~K}$ with a regulator which feeds a heater attached to the sample. The relative stability of the temperatures obtained is better than $10^{-5}$.

At equilibrium the power provided to the sample is equal to the constant power flowing to the bath. Any power provided or absorbed by the sample is thus detected through a variation of the regulating power. For a time constant of the thermal link of $0.35 \mathrm{~s}$ the sensitivity of our detection is $0.01 \mu \mathrm{W}$ over measurement times between $10 \mathrm{~s}$ and $10^{4} \mathrm{~s}$.

3. Preliminary results. - We have observed an energy relaxation, at constant temperature and in zero field, from a sample of $\underline{\mathrm{AuFe}} 4$ at. \% (weight $10 \mathrm{~g}$ ) in all circumstances where the sample exhibits a remanent magnetization (in all cases the energy flow was from the sample to the bath). If, on the contrary, the sample is cooled below $T_{\mathrm{g}}(\sim 20 \mathrm{~K})$ in zero field and maintained in zero field, no measurable relaxation is observed. This is somewhat in contrast with what is observed in a glass or in a polymer where energy relaxations (of both signs) are observed in the vicinity of the glass temperature under the influence of purely thermal cycling.

We have checked that no measurable relaxation is observed on a sample of pure gold containing less than 4 at. p.p.m. of magnetic impurities [4] whatever the thermal and magnetic path followed to reach the experimental conditions. The results which we 
will discuss below are therefore characteristic of the spin glass phase.

4. Results. - The results which we present here were obtained in the limiting case where the remanent magnetization is saturated. In practice the sample is cooled from $30 \mathrm{~K}$ in a magnetic field of $25 \mathrm{kOe}$ (sufficient to saturate the thermoremanent magnetization) down to the temperature of the bath $(1.4 \mathrm{~K})$. The magnetic field is then removed in a few minutes, taking care that the induced eddy currents do not cause any sizeable elevation of the temperature.

A first measurement of the energy flux $\dot{Q}$ provided by the sample is then made vs. time with the temperature maintained at $1.62 \mathrm{~K}$. After long times (of the order of $600 \mathrm{~s}$ ) have elapsed, the power provided becomes comparable to the sensitivity of our apparatus. If the temperature of the sample is then increased, the energy flow again becomes measurable.

Figure 1 shows the data which we have obtained at different temperatures. $\dot{Q}^{-1}$ is shown $v s$. the time of measurement. This diagram demonstrates the dominant $t^{-1}$ behaviour of the energy relaxations, a feature which has been previously reported [5]. However, deviations from this behaviour are observed at higher temperatures for long times.

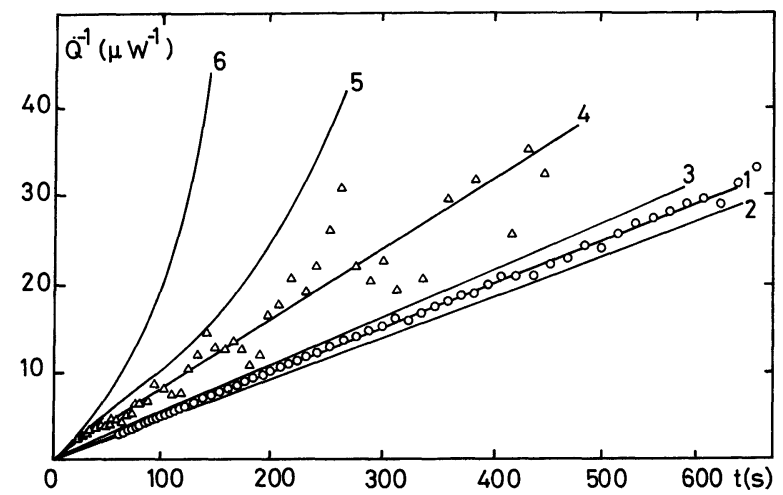

Fig. 1. - Time dependence of reciprocal power delivered by a $10 \mathrm{~g} \mathrm{AuFe} 4 \%$ sample at various temperature : (1) $1.65 \mathrm{~K}$, (2) $1.87 \mathrm{~K}$, (3) $2.34 \mathrm{~K}$, (4) $2.98 \mathrm{~K}$, (5) $3.3 \mathrm{~K}$, (6) $4.15 \mathrm{~K}$. Curves are a smooth fit of experimental data points. For clarity only two characteristic sets of data are plotted : curve (1) (O) low experimental scattering level, curve $2(\Delta)$ high experimental scattering level.

Figure 2 shows the power $\dot{Q}$ provided by the sample at fixed time $(60 \mathrm{~s})$ for the different temperatures of relaxation. A maximum is observed around $1.87 \mathrm{~K}$.

These results show very clearly that a slow energy relaxation is associated with the well-known relaxation of the remanent magnetization.

5. Interpretation. - One of the characteristic features of disordered systems (glasses, polymers, spin glasses, ...) is their ability to remain out of thermodynamic equilibrium for unusually long times.

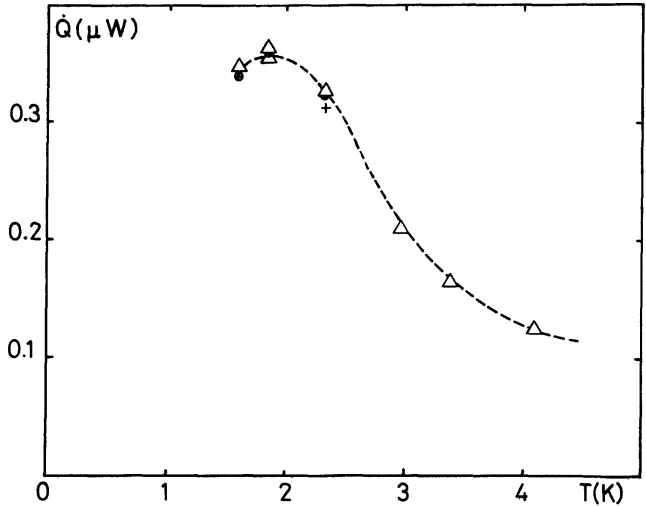

Fig. 2. - Temperature dependence of the power delivered by a $10 \mathrm{~g} \mathrm{AuFe} 4 \%$ sample at $t=60 \mathrm{~s}$. C exp. № $1, \triangle \exp$. № $2,+\exp$. No 3 , exp. No 4 .

In the absence of any satisfactory microscopic description, phenomenological models provide simple but effective schemes which can guide our understanding of the experimental results. This type of model recognizes the existence of objects [6] (two level systems [7], magnetic clouds [8], fine grains [9], clusters...) characterized by two independent random variables (a potential barrier $V$ and a splitting $W$ ) distributed over large energy scales.

In our experiment, the spin glass system has acquired some energy by following a thermomagnetic path $\mathrm{C}(H(t), T(t))$ (characteristic of the history through which the sample has gone to reach the experimental conditions) and we observe the time variation of this energy at a given fixed temperature $T$ in zero field.

Assuming thermally activated processes, the relaxation time associated with a barrier of height $V$ is given by the Arrhenius law :

$$
\tau=\tau_{0} \exp \left(V / k_{\mathrm{B}} T\right)
$$

$\tau_{0}$ is a relaxation time characteristic of the system.

The measurement time $t$ introduces a cut off in the barrier heights; we have :

$$
V_{\mathrm{c}}=k_{\mathrm{B}} T \ln \frac{t}{\tau_{0}}
$$

The objects with a barrier height $V>V_{\mathrm{c}}$ are not observable on the time scale $t$. The time $t$, therefore, will appear in the expression of the energy (or of the magnetization...) through the occurrence of this cut off $V_{\mathrm{c}}$. In particular the excess energy stored in the system at the temperature $T$ in zero field, is only a function of the thermomagnetic path followed and of the cut off $V_{c}$ and can thus be expressed as :

$$
Q=f\left(\mathrm{C}, k_{\mathrm{B}} T \ln \frac{t}{\tau_{0}}\right) \text {. }
$$

The energy flux $\dot{Q}$ is then :

$$
\dot{Q} \equiv \frac{\partial Q}{\partial t}=\frac{k_{\mathrm{B}} T}{t} f^{\prime}\left(\mathcal{C}, k_{\mathrm{B}} T \ln t / \tau_{0}\right) .
$$


In practice $\ln t / \tau_{0} \sim 15$ in spin glasses, and depends only slightly on $t$ for reasonable measuring times [2]. Therefore at constant temperature, the evolution of $\dot{Q} v s$. $t$ should be mainly like $t^{-1}$ in agreement with the experimental data at low temperatures (Fig. 1). More precisely $\frac{t \dot{Q}}{k_{\mathrm{B}} T}$ is a universal function of $k_{\mathrm{B}} T \ln \frac{t}{\tau_{0}}$ for a given thermomagnetic path. This general argument follows from the Arrhenius law (1) and implies the presence of thermally activated objects. Figure 3 shows $t \dot{Q} / T$ at $60 \mathrm{~s}$ from our data. A dominant exponential behaviour is observed which is reminiscent of the behaviour of the corresponding saturated remanent magnetization [8].

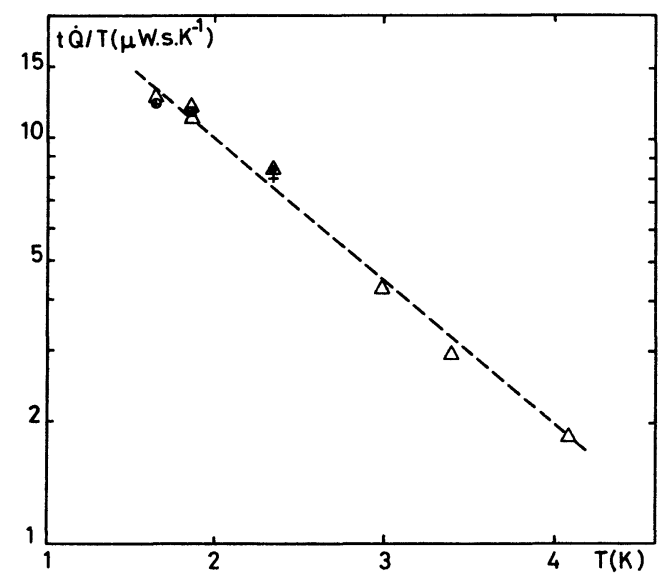

Fig. 3. - Temperature dependence of $t \dot{Q} / T$ for a $10 \mathrm{~g} \underline{\mathrm{AuFe}} 4 \%$ sample for $t=60 \mathrm{~s}$. $O$ exp. № $1, \Delta \exp$. № $2,+$ exp. № 3 , $\bullet$ exp. No 4.

In order to obtain an accurate estimation of $\dot{Q}$ it is necessary to make some further assumptions (about the origin of the objects and the distributions of $V$ and $W$, etc...).

Different schemes are possible which lead to similar results. In spin glasses three parameters characterize an object : the barriers height $V$, the splitting $W$ and the magnetic moment $M g$ (in the model of magnetic clouds [8], $V$ and $M g$ were expressed in terms of a unique parameter $n$, the number of spins contained in a cloud, which is assumed to obey a well defined distribution).

Taking $V, W$ and $M g$ as random variables and assuming that the distribution $P(W)$ depends only slightly on $W$ over a width larger than that of the distribution $P(V)$ [10], we obtain the following result :

$$
\dot{Q} \simeq \frac{k_{\mathrm{B}} T}{t} \frac{M_{\mathrm{R}}}{\overline{M g}}
$$

where $\overline{M g}$ is the average moment of the objects and $M_{\mathrm{R}}$ is the saturated remanent magnetization.

It is possible to account for the existence of a remanent magnetization by only the presence of potential barrier (symmetrical double well potential). However, to account for the existence of $\dot{Q}$ it is necessary to associate a splitting with these barriers. Our results show the existence of a large distribution of these splittings. In its present form, the model contains the same ingredients used in the models which describe the vitreous state $[6,7]$. This similarity in the low energy excitations (which shows up as a distribution $P(W)$ which is flat on the scale $\left.k_{\mathrm{B}} T\right)$ seems thus to be a characteristic of disordered systems.

In practice the saturated remanent magnetization $M_{\mathrm{R}}$ is known to be experimentally well described by a law of the form $[2,11,8]$.

$$
M_{\mathrm{R}}=M_{0} \exp \left(-\frac{T}{T_{0}} \ln \frac{t}{\tau_{0}}\right) \equiv M_{0}\left(\frac{t}{\tau_{0}}\right)^{-T / T_{0}} .
$$

Using (5) and (6) we get :

$$
M_{\mathrm{R}}=M_{0} \exp \left(-\frac{T}{T_{\mathrm{m}}}\right)=\frac{t \dot{Q}}{k_{\mathrm{B}} T} \overline{M g}
$$

where we have written $T_{0} / \ln \frac{t}{\tau_{0}}=T_{\mathrm{m}}$.

From eq. (7) it is easily seen that $\dot{Q}(T)$ measured at time $t$ goes through a maximum at $T_{\mathrm{m}}$, and that $t \dot{Q} / T$ is an exponential function of the temperature, two features which are illustrated on figures 2 and 3 respectively. Preliminary measurements of the relaxations of the remanent magnetization of the same sample in the same conditions of field, time and temperature reveal a very good agreement with the predictions of eq. (7) over the whole range of temperature [12]. We find for the average magnetization of an object $\overline{M g} \sim 95 \mu_{\mathrm{B}}$ in good agreement with other independent estimations [2, 13]. In addition it is interesting to note that Monte-Carlo simulations in a three dimensional spin glass [14] gave an energy decay towards equilibrium (starting from ferromagnetic alignment) varying like $t^{-a}$ with the exponent $a$ being the same as for the magnetization which is consistent with our eq. (7). Furthermore the value $a \sim T / 2$ which they estimate at $T_{\mathrm{G}}$ would be consistent with our result $\left(a \sim T / T_{0}\right.$ with $\left.T_{0} \sim 49 \mathrm{~K}\right)$ in as much as it is possible to extrapolate the present data up to $T_{\mathrm{G}} \sim 20 \mathrm{~K}$.

6. Conclusion. - We have shown the existence of an energy relaxation associated with the relaxation of the remanent magnetization in a spin glass. Our analysis has allowed us to justify the presence of thermally activated objects with a large distribution of low energy excitations. We thus established the pertinence of a model which seems of very general validity for the description of disordered substances. However, the following differences should be kept in mind :

- in spin glasses, thermally activated processes 
dominate at low temperatures while tunnelling states are dominant in glassy glasses;

- close to the glass transition where thermally activated processes dominate in glasses and in polymers, the distribution of relaxation times is much narrower. In our case the spectrum is very broad because of the very wide distributions of both the barrier heights and the splittings involved.

Acknowledgments. - The authors wish to acknowledge the technical assistance of Miss A. Dufresne and MM. P. Brosse-Maron and D. Tomasik.

\section{References}

[1] Blandin, A., J. Physique Colloq. 39 (1978) C6-1499. Souletie, J., J. Physique Colloq. 39 (1978) C2-3.

[2] Tholence, J. L., Thèse d'Etat, Université de Grenoble (1973). TOURNIER, R., Thèse, Université de Grenoble (1965).

[3] Kovacs, A. J., Fortschr. Hochpolym. Forsch. 3 (1963) 394. Kovacs, A. J., Rheol. Acta 5 (1966) 262.

Bourdariat, J., Berton, A., Chaussy, J., ISNARd, R., Odin, J., Polymer 14 (1973) 167.

[4] Tissier, B., Private communication.

[5] Nieuwenhuys, G. J. and Mydosh, J. A., Physica 86-88B (1977) 880.

[6] Rammal, R., Thèse, Université de Grenoble (1977).

[7] Anderson, P. W., Halperin, B. I., Varma, C. M., Philos. Mag. 25-1 (1972).

Philips, W. A., J. Low Temp. Phys. 7 (1972) 351.

[8] Holtzberg, F., Tholence, J. L., Tournier, R., Proceedings 2nd Symposium on Amorphous Magnetism, N. Y., Aug. 2527, 1976.
[9] NéEL, L., Ann. Géophys. 5 (1949) 99.

NÉEL, L., J. Phys. Soc. Japan 17B1 (1962) 676.

[10] Prejean, J. J., Souletie, J., to be published.

[11] Prejean, J. J., J. Physique Colloq. 39 (1978) C6-907.

[12] Berton, A., Chaussy, J., Odin, J., Prejean, J. J., Rammal, R., SOUleTIE, J. and TourniER, R., to be published. The $T$ dependence measured here happens to be markedly different from what would be expected by extrapolating previous data of the saturated TRM [2] of lower concentration. Notice however that the procedure is different from that in ref. [2] where the sample had been thermocooled at each temperature. Furthermore the field we have used $(25 \mathrm{kOe})$ is probably not sufficient to fully saturate the TRM. Finally the sample we used in the present experiment has been aged one year at room temperature.

[13] Canella, V., Mydosh, J., Phys. Rev. B 6 (1972) 4220

[14] Binder, K., Stauffer, R., Phys. Lett. 57A (1976) 177. 